\title{
Predictors of Neurofeedback Outcomes Following qEEG Individualized Protocols for Anxiety
}

\author{
J. Claire Gregory*, Devon E. Romero, and Mark S. Jones
}

Department of Counseling, University of Texas at San Antonio, San Antonio, Texas, USA

\begin{tabular}{|c|c|}
\hline \multicolumn{2}{|c|}{ Abstract } \\
\hline \multicolumn{2}{|c|}{$\begin{array}{l}\text { In this retrospective study, researchers examined effects of quantitative electroencephalography (qEEG), } \\
\text { individualized neurofeedback treatment protocols for anxiety. The present study includes } 52 \text { clients with } 53.8 \% \\
\text { ( } n=28 \text { ) self-reporting as male and included two time points (pre and post). Secondary analyses utilized a subset } \\
\text { of client data }(n=21 \text { ) with measurements from three time points (pre, post, and follow-up). All clients completed } \\
\text { qEEG and self-report assessments. Clients agreed to attend a minimum of } 15 \text { biweekly sessions, for one } \\
\text { academic semester. Findings from regression analyses revealed three predictors of posttreatment outcomes. } \\
\text { In addition, analysis of a subsample of data assessed at three time points revealed statistically significant } \\
\text { improvement from pre to post and sustained outcomes from post to follow-up. We discuss limitations and } \\
\text { implications for future research. }\end{array}$} \\
\hline \multicolumn{2}{|c|}{ Keywords: neurofeedback; anxiety; qEEG-guided amplitude neurofeedback; predictor } \\
\hline \multicolumn{2}{|c|}{$\begin{array}{l}\text { Citation: Gregory, J. C., Romero, D. E., \& Jones, M. S. (2020). Predictors of neurofeedback outcomes following qEEG individualized } \\
\text { protocols for anxiety. NeuroRegulation, } 7(1), 18-25 . \text { https://doi.org/10.15540/nr.7.1.18 }\end{array}$} \\
\hline $\begin{array}{l}\text { *Address correspondence to: J. Claire Gregory, University of } \\
\text { Texas at San Antonio, Department of Counseling, 501 W. Cesar E. } \\
\text { Chavez, San Antonio, TX 78207, USA. Email: } \\
\text { jessica.gregory@utsa.edu }\end{array}$ & $\begin{array}{l}\text { Edited by: } \\
\text { Rex L. Cannon, PhD, SPESA Research Institute, Knoxville, } \\
\text { Tennessee, USA }\end{array}$ \\
\hline $\begin{array}{l}\text { Copyright: @ 2020. Gregory et al. This is an Open Access article } \\
\text { distributed under the terms of the Creative Commons Attribution } \\
\text { License (CC-BY). }\end{array}$ & $\begin{array}{l}\text { Reviewed by: } \\
\text { Rex L. Cannon, PhD, SPESA Research Institute, Knoxville, } \\
\text { Tennessee, USA } \\
\text { Randall Lyle, PhD, Mount Mercy University, Cedar Rapids, lowa, } \\
\text { USA }\end{array}$ \\
\hline
\end{tabular}

Although many Americans experience stress during their lifetime, anxiety disorders can be debilitating and disrupt daily functioning. Anxiety disorders are a pervasive and prevalent mental health concern affecting $19.1 \%$ of adults in the United States (National Institute of Mental Health [NIMH], 2017). In addition, an estimated $31.1 \%$ of adults in the United States experience an anxiety disorder at some point during their lives (NIMH, 2017). Although various anxiety disorders exist, prevalent ones are generalized anxiety disorder, panic disorder, and several phobia-related disorders (NIMH, 2018).

Frequently, the onset of anxiety disorders begins during childhood and, if an individual does not receive treatment, anxiety symptoms may persist throughout their life (American Psychiatric Association [APA], 2013). Symptoms of anxiety differ from person to person and can include a range of physiological and psychological issues. For example, people with generalized anxiety disorder can experience muscle tension, trouble concentrating, and difficulty controlling thoughts of worry; while people with panic disorder experience unexpected panic attacks, accelerated heart rate, and feeling out of control (NIMH, 2018). Although symptoms can vary from one type to another, the Diagnostic and Statistical Manual of Mental Disorders (5th ed.; DSM-5) suggests that anxiety disorders share commonalities of "excessive fear and anxiety and related behavioral disturbances" (APA, 2013, p. 189).

\section{Literature Review}

Clinicians display use of various forms of biofeedback modalities for treating anxiety (Jones \& Hitsman, 2018). Some biofeedback modalities include heart rate variability (HRV) training and electromyography (EMG). Neurofeedback is also a form of biofeedback that is showing promising effects in correcting negative symptoms including depression (Cheon et al., 2015), sleep disorders (Cheon et al., 2015), attention-deficit/hyperactivity disorder (Van Doren et al., 2019), and anxiety (Cheon et al., 2015; Kerson, 
Sherman, \& Kozlowski, 2009; Scheinost et al., 2013; Wang et al., 2019; Zhao et al., 2018). In addition, combinations of neurofeedback and biofeedback (e.g., HRV) have produced promising outcomes for reducing anxiety symptoms (White et al., 2017).

In 2015, Cheon et al. decided to conduct a controlled study focusing on psychiatric patients and neurofeedback. They used retrospective data, and the data collection process included the administration of the Clinical Global ImpressionSeverity scale (CGI-S; Busner \& Targum, 2007) and the Hill-Castro Checklists (Hill \& Castro, 2002) on a weekly basis. The CGI is a widely known and utilized tool for objective rating (Cheon et al., 2015) and $23.4 \%$ of their participants had an anxiety disorder recognized by the $D S M-5$. All patients participated in beta, SMR, or alpha/theta neurofeedback training at training sites: Fp1, Fp2, F3, F4, F7. F8, T3, T4, C3, C4, P1, P2, O1, O2, and Oz according to the 10-20 Electrode system. The researchers identified varying diagnoses as rationale for using unique neurofeedback protocols. In addition, the authors state that during the final neurofeedback sessions, protocol development shifted to fit individual needs (Cheon et al., 2015). Both the CGI-S and the HillCastro Checklist resulted in significant findings ( $p$ $=.0001)$ for the anxiety participants.

Numerous other researchers found evidence of neurofeedback being beneficial for anxiety symptoms. Researchers Scheinost et al. (2013) sought to examine state verses trait anxiety and alpha asymmetry while using real-time functional magnetic resonance imaging (rt-fMRI). They discovered that focusing training on brain regions instead of certain brain sites was advantageous. The participants also showed lasting results for emotion regulation and decreased anxiety. In addition, proponents of a study by Zhao et al. (2019) displayed a reduction of anxiety symptoms lasting 3 days when using rt-fMRI. Drawing on work from previous studies, Zhao et al. (2019) emphasized training on connectivity of individual pathways of participants' amygdalaprefrontal area. Therefore, neurofeedback protocols based on individualized protocols and/or variations in EEG data, show promising results for improving emotion regulation and decreasing anxiety.

Each study has certain strengths and limitations and variations exist among neurofeedback training modalities and protocols. One limitation is using the same protocol for each patient, and another is utilizing only symptom-based protocols. According to Hammond (2010), it is important to assess a baseline quantitative electroencephalography (qEEG) pattern as this will help identify the heterogeneity in brain wave patterns, find comorbidities, and examine the brain for medication effects. Viewpoints for neurofeedback clinicians still vary on whether to use individualized protocols or the same protocol. Some researchers view individualized protocols as a strength. Agreeing with this viewpoint, Arns, Heinrich, and Strehl (2014) suggest that researchers may want to consider using individualized protocols. Certain researchers postulate the benefits of using individualized protocols and how they can tailor neurofeedback to every person's unique brain patterns (Arns et al., 2014; Hammond, 2010; Krigbaum \& Wigton, 2014).

An individualized protocol consists of tailoring treatment to a person's specific qEEG data. This neurofeedback modality dates to the 1980s (Krigbaum \& Wigton, 2014). Currently, many studies indicate the benefits of personalized protocols (Arns, Drinkenburg, \& Kenemans, 2012; Ogrim \& Hestad, 2013; Walker, 2012). Specifically, individualized protocols allow the clinician to determine client-based protocols which also take into consideration the diversity of qEEG patterns (Hammond, 2010).

In an additional study by Wigton and Krigbaum (2015), the researchers further assert how $z$-score protocols (e.g., individualized protocols) aid in identifying the link between specific cortical dysfunctions and connectivity concerns related to mental health symptomology. Moreover, to determine neurofeedback training sessions, the comparison of qEEG data to a normative database results in z-scores (Wigton \& Krigbaum, 2015). This method allows for a baseline of pretreatment data for determining the progress of the client. With this knowledge, clinicians can reduce mental health issues by bringing the scores closer to the mean.

Data collection for this retrospective study consisted of methods inspired by $z$-score training. Since the current study's data was collected from a student training clinic, the neurofeedback clinic director decided on single-channel amplitude training for three reasons: (a) this training is commonly used by clinicians, (b) it is an easier starting point for students in training versus more advanced modalities, and (c) numerous one-channel amplitude training research literature is reviewed by Wigton (2014; Jones \& Hitsman, 2018). Therefore, the retrospective data included in this study were examining reduction of anxiety symptoms while utilizing qEEG-guided amplitude neurofeedback training protocols. 
The present study uses deidentified data collected from a graduate training counseling center with the primary aim of training master's- and doctoral-level counseling students interested in developing clinical neurofeedback competency. The purpose of this study is to examine predictors of neurofeedback outcomes following qEEG individualized protocols for treating anxiety. Specifically, the study aimed to answer the following question: "Are there differences within the individual that predict improvement in client-reported anxiety symptoms following individualized neurofeedback treatment?" Secondary research questions include:

- "Does individual neurofeedback treatment differ in participant self-report of anxiety concerns over time?"

- "Does individual neurofeedback treatment differ from participant self-report from pretest to follow-up?"

- "Does individual neurofeedback treatment differ from participant self-report from pretest to posttest?", and

- "Does individual neurofeedback treatment differ from participant self-report from posttest to follow-up?"

\section{Method}

\section{Participants/Sample/Power}

The University of Texas at San Antonio (UTSA) Institutional Review Board deemed this retrospective study exempt from review. Potential clients contacted Sarabia Family Counseling Center at the University of Texas at San Antonio (UTSA) to inquire about neurofeedback treatment for anxiety. Upon calling, a master's- or doctoral-level student in UTSA's Counseling Department screened clients to determine their eligibility for treatment. This screening process included inclusion criteria of reporting primarily anxiety symptoms, availability, and meeting the age requirements. Clients enrolled in the neurofeedback treatment program agreed to attend a minimum of 15 neurofeedback sessions biweekly, which were free of charge.

The present study consists of a sample of 52 clients aged 19 to $59(M=36.4, S D=12.6)$. Of these clients, $53.8 \%(n=28)$ self-reported as male. Ethnic composition of the clients included $50 \%(n=26)$ NonHispanic, 44\% $(n=23)$ Hispanic/Latino, and 6\% $(n=$ 3) did not respond. Table 1 provides a more detailed review of client demographics.

\begin{tabular}{|c|c|}
\hline $\begin{array}{l}\text { Table } 1 \\
\text { Characteristics of the sample at time } \\
(N=52) \text {. }\end{array}$ & of recruitment \\
\hline Mean age (range) & $36.37(19-59)$ \\
\hline Gender & \\
\hline Male & $53.8 \%$ \\
\hline Female & $42.3 \%$ \\
\hline Chose not to respond & $3.9 \%$ \\
\hline Ethnicity & \\
\hline Non-Hispanic & $50.0 \%$ \\
\hline Hispanic/Latino & $44.2 \%$ \\
\hline Did not respond & $5.8 \%$ \\
\hline Education & \\
\hline No HS diploma; no GED & $1.9 \%$ \\
\hline GED & $1.9 \%$ \\
\hline High school degree & $9.6 \%$ \\
\hline Some college; no degree & $28.8 \%$ \\
\hline Associate degree & $9.6 \%$ \\
\hline Bachelor's degree; RN & $23.1 \%$ \\
\hline Some grad school; no degree & $9.6 \%$ \\
\hline Master's degree & $7.7 \%$ \\
\hline PhD; Law degree & $3.9 \%$ \\
\hline Did not respond & $3.9 \%$ \\
\hline
\end{tabular}

Secondary analyses utilized a subsample of the dataset described above. This subsample includes 21 clients with assessments completed at three time points (pre, post, and follow-up). In terms of demographic data of the subsample, clients' $(n=21)$ ages range from 20 to $56(M=38.8, S D=12.39)$ with $61.9 \%(n=13)$ of clients self-reporting as female. The self-reported ethnic composition of the subsample was $38.1 \%(n=8)$ Non-Hispanic, $52.4 \%$ $(n=11)$ Hispanic/Latino, and $9.5 \%(n=2)$ chose not to respond.

\section{Clinicians}

Clinicians for the study included student clinicians which were clinical mental health master's-level students and counselor education and supervision doctoral-level students. Before beginning their neurofeedback sessions, the students previously completed the Biofeedback Certification International Alliance requirements for didactic coursework for 
neurofeedback. In addition, student clinicians are supervised by a certified and licensed supervisor. At times, trained volunteer clinicians (e.g., faculty, alumni, etc.) served as clinicians.

\section{Measures}

Demographic information and treatment record. Demographic data for this study includes age, gender, highest level of education completed, ethnicity, and previous or current experience with counseling. Additional data collected consists of number of attended sessions, session-to-session records, type of protocol, amplitude measures for each frequency band, and electrode placement.

Zung self-rating anxiety scale for adults. The Zung Self-rating Anxiety Scale (SAS) is a 20-item, Likert-type, self-report measure of state and trait anxiety based on cognitive, autonomic, motor, and central nervous system symptoms manifestations. Example items include "My face gets hot and blushes," "I have nightmares," and "I feel afraid for no reason at all." With raw scores ranging from 20 to 80, higher scores indicate greater severity of anxiety symptomatology (Zung, 1971). The SAS has demonstrated good internal consistency with a Cronbach's alpha of .82 (Tanaka-Matsumi \& Kameoka, 1986).

Self-report for the Achenbach system of empirically based assessment. The Adult SelfReport (ASR) is part of the Achenbach System of Empirically Based Assessment (Achenbach \& Rescorla, 2003). The ASR is a 120-item, Likert-type, self-report measure that assesses maladaptive behavioral and emotional problems. The ASR is appropriate for adults between the ages of 18 to 59 years. The ASR consists of adaptive functioning, syndrome, DSM-oriented, and substance use scales and has demonstrated good test-retest reliability (Education, $r=.80$; Mean Substance Use, $r=.96$; Achenbach \& Rescorla, 2003) and internal consistency for scales utilized in the present study (Total Problems, $\alpha=.97$; Achenbach \& Rescorla, 2003).

\section{Instrumentation}

Quantitative electroencephalography. The research team instructed participants to limit consumption of nonessential substances 24 hours prior to the qEEG recording. However, the research team factored medically required substances into the qEEG interpretation and subsequent protocol development. The collection of qEEG data occurred with a 19-channel recording using a BrainMaster
Discovery 24 (BrainMaster Technologies, Inc., Bedford, OH) high-impedance amplifier. The software utilized was NeuroGuide (Applied Neuroscience, Inc., Largo, FL) which included 5 min of eyes open (EO) and 5 min of eyes closed (EC). Clients' qEEG recordings included fittings for the correct size of Electro-Cap (Electro-Cap International, Inc., Eaton, $\mathrm{OH}$ ) 10-20 electrode placement with impedance levels less than $5 \mathrm{k} \Omega$. Preparation for the qEEG also included cleaning the ground and reference locations with abrading $\mathrm{PCl}$ prep pads, Nuprep skin prep gel, and rubbing alcohol (Jones \& Hitsman, 2018). A member of the research team used the resulting data to develop an individualized protocol for anxiety.

Neurofeedback. For the neurofeedback sessions, clinicians used the BrainMaster Atlantis two-channel amplifiers and BioExplorer (Cyberevolution, Inc., Seattle, WA) software. When preparing the electrode sites, clinicians cleaned the skin with rubbing alcohol and used abrading $\mathrm{PCl}$ prep pads when needed for ground and reference locations. Clinicians used Nuprep to help impedance levels and Ten20 conductive paste to attached gold-plated electrodes to the client's scalp. During the sessions, clinicians monitored impedance measurements to ensure that interelectrode impedance was less than $5 \mathrm{k} \Omega$ (Jones, 2015).

\section{Neurofeedback Protocols}

The research team instructed participants to discontinue the consumption of caffeine or other nonessential substances on neurofeedback days. Range of attended sessions were 3-23 ( $M=13.4, S D$ $=4.3)$ for the primary data set, and $3-20(M=13, S D$ $=4.87)$ for the secondary data set. Clinicians provided neurofeedback using BrainMaster Atlantis two-channel amplifiers and BioExplorer software. Training protocols included amplitude uptraining and/or downtraining of preferred frequency bands based on qEEG results. Further, protocol selections were influenced by current research and reflect markers thought to be associated with anxiety concerns (Demerdzieva \& Pop-Jordanova, 2011; Gunkelman, 2006; Heller, Nitschke, Etienne, \& Miller, 1997; Price \& Budzynski, 2009; Stern, 2005, p. 196; Tharawadeepimuk \& Wongsawat, 2014). For example, one client's protocol consisted of EO CZ downtraining 4-9 Hz, uptraining $12-15 \mathrm{~Hz}$, and downtraining $17-23 \mathrm{~Hz}$. Another example of a client's protocol was EC $\mathrm{PZ}$ downtraining $3-7 \mathrm{~Hz}$, uptraining 8-10 Hz, and downtraining $25-30 \mathrm{~Hz}$. 
According to preferences of participants and clinicians' clinical judgment, feedback was determined using the following formats: animations, sounds, games, and analog presentations. The predetermined thresholds were set manually at the start of the session with an ideal reward rate of $50 \%$. During the sessions, clinicians made periodic adjustments to the threshold settings as an attempt to share behavior toward the client's treatment goals. Treatment records where kept for each session and included frequency bands, threshold settings, average amplitude, type of feedback received, and any other clinician notes. Training sessions lasted approximately 20 minutes.

\section{Statistical Analysis}

Statistical analyses for this study included the Statistical Package for the Social Sciences (SPSS) software version 25 (SPSS, 2017). Missing data, examining cases for missing data, outliers, and normality were all assessed before analysis of data. Analytic computations used $p$-values set at $\alpha=0.05$. The primary data set has $0 \%$ missing data for the SAS, and $5.8 \%$ for the ASR. For the subsample data set, there was no missing data.

The research team ran regression analyses on the primary data set to determine potential predictors of post-SAS scores. We controlled for pre-SAS scores and client-reported gender due to high correlation with the outcome variable (post-SAS scores). Education was dummy coded 0 (no) and 1 (yes) with No high school (HS) diploma serving as the reference group. The present study utilized control variables and predictor variables measured by the ASR at time 1. Additionally, using the subsample data set, researchers used a paired samples $t$-test to measure mean differences between pre, post, and follow-up scores of the SAS.

\section{Results}

Individuals completed the SAS and ASR at intake (time 1, pre) to assess their level of anxiety and other concerns. Upon completion of the neurofeedback treatment protocols, both assessments were readministered (time 2, post). The mean prescore results from all subjects was $45.62(S D=8.49)$, while the mean for the postscores was $39.50(S D=9.40)$.

A regression analysis displayed total problems as measured by the ASR significantly predicted postSAS scores $(B=.26$, SE $B=.24, p=.05)$. This model explained $56 \%$ of the total variance after controlling for pre-SAS scores and gender; $F(3,48)=21.13, R^{2}$ $=.56, p<.01$. This demonstrated a significant positive relationship between total problems and post-SAS scores, showing greater improvement in anxiety symptoms following neurofeedback treatment was associated with lower total problems scores as measured by the ASR prior to neurofeedback treatment.

An additional regression analysis revealed that a model including gender, pre-SAS scores, and mean substance use predicted post-SAS scores; $F(3,48)=$ $21.74, R^{2}=.58, p<.01$. Mean substance use was a significant predictor in this model and explained $58 \%$ of the total variance after controlling for pre-SAS scores and gender. This demonstrated a significant positive relationship between mean substance use and post-SAS scores, showing greater improvement in anxiety symptoms following neurofeedback treatment was associated with lower mean substance use scores as measured by the ASR prior to neurofeedback treatment.

A third regression analysis identified some college with no degree $(B=15.84, S E B=.7 .18, p=.03)$, bachelor's degree $(B=17.11$, SE $B=7.22, p=.02)$, and $\mathrm{PhD}$ or law degree $(B=19.28$, SE $B=8.69, p$ $=.03$ ) significantly predicted post-SAS scores when controlling for pre-SAS and gender; $F(10,39)=6.45$, $R^{2}=.62, p<.01$. This demonstrated a significant positive relationship between education and postSAS scores, showing higher education was associated with greater improvement in anxiety symptoms following neurofeedback treatment. Regression results for primary analyses appear in Table 2. 


\section{Table 2}

Regression analysis summary for variables found to predict post-SAS scores $(N=52)$.

\begin{tabular}{lrrr}
\hline & \multicolumn{1}{c}{$B$} & SE $B$ & \multicolumn{1}{l}{$t$} \\
$F(3,48)=21.13, R^{2}=.57, p<.01$ & & \\
Gender & 1.10 & 1.77 & 0.62 \\
Pre-SAS scores & 0.70 & 0.15 & $4.856^{\star \star}$ \\
Total problems & 0.256 & 0.125 & $2.038^{\star}$ \\
$F(3,48)=21.74, R^{2}=.58, p<.01$ & & \\
Gender & 1.83 & 1.72 & 1.06 \\
Pre-SAS scores & 0.91 & 0.12 & $7.9^{\star \star}$ \\
Mean substance use & -0.341 & 0.153 & $-2.237^{\star}$ \\
$F\left(10\right.$, 39) $=6.45, R^{2}=.62, p<.01$ & & \\
Gender & 4.06 & 2.03 & $1.99^{\star}$ \\
Pre-SAS scores & 0.91 & 0.13 & $7.08^{\star \star}$ \\
GED & 15.19 & 9.95 & 1.53 \\
High school degree & 12.47 & 7.58 & 1.64 \\
Some college; no degree & 15.84 & 7.18 & $2.21^{\star}$ \\
Associate degree & 9.42 & 7.53 & 1.25 \\
Bachelor's degree; RN & 17.11 & 7.22 & $2.37^{\star}$ \\
Some grad school; & & & \\
no degree & 13.72 & 7.62 & $1.8^{\dagger}$ \\
Master's degree & 10.75 & 7.77 & 1.38 \\
PhD; Law degree & 19.28 & 8.69 & $2.22^{\star}$ \\
\hline
\end{tabular}

Secondary analyses using the subsample data included a third time point (time 3 , follow-up). This time point occurred one month after posttreatment. The mean of the prescores was $45.67(S D=9.34)$, mean postscores was $39.14(S D=9.39)$, and mean follow-up scores was $41.05(S D=9.58)$. We ran paired sample $t$-tests to examine differences between time points of the SAS scores (time 1, time 2, and time $3)$. Results displayed statistically significant change from pre to post $t(20)=4.7, p<.001, d=.68$, and from pre to follow-up $t(20)=2.66, p=.015, d=.47$. There was no significant change from post to followup $t(20)=-1.67, p=.111, d=.20$. Results appear in Table 3 and Figure 1 illustrates these findings.

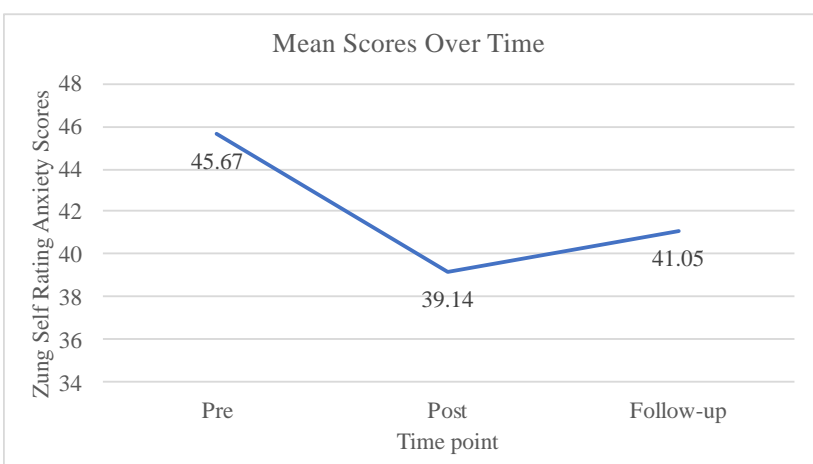

Figure 1. Zung Self-rating Anxiety Scale mean scores at pre, post, and follow-up $(n=21)$.

Note. ${ }^{\dagger} p<.10 ;{ }^{*} p<.05 ;{ }^{* *} p<.01$

\section{Table 3}

Zung Self-rating Anxiety Scale (SAS) for adults.

\begin{tabular}{|c|c|c|c|c|c|c|c|}
\hline & \multicolumn{2}{|c|}{ Time Point } & \multicolumn{2}{|c|}{ Change } & \multirow[b]{2}{*}{$t(d f)$} & \multirow[b]{2}{*}{$p$} & \multirow[b]{2}{*}{$d$} \\
\hline & $M(S D)$ & $M(S D)$ & $M(S D)$ & {$[95 \% \mathrm{Cl}]$} & & & \\
\hline & Pre & Post & & & & & \\
\hline \multirow[t]{2}{*}{ Time 1 - Time 2} & 45.67 (9.94) & 39.14 (9.38) & $6.52(6.37)$ & {$[3.63,9.42]$} & $4.7(20)$ & $<.001$ & .68 \\
\hline & Post & Follow-up & & & & & \\
\hline \multirow[t]{2}{*}{ Time 2 - Time 3} & $39.14(9.38)$ & 41.05 (9.58) & $-1.905(1.14)$ & {$[-4.28, .478]$} & $-1.67(20)$ & .111 & .20 \\
\hline & Pre & Follow-up & & & & & \\
\hline Time 1 - Time 3 & $45.67(9.94)$ & 41.05 (9.58) & $4.62(7.94)$ & {$[1.01,8.23]$} & $2.66(20)$ & .015 & .47 \\
\hline
\end{tabular}




\section{Discussion}

The primary aim of this retrospective study was to examine predictors of anxiety symptom outcomes following individualized neurofeedback treatment. The present study identified a large mean decrease in SAS outcomes from pretreatment to posttreatment, indicating improvement in client-reported anxiety symptoms following neurofeedback treatment. Regression analyses identified total problems, substance use, and level of education as predictors of anxiety symptoms as measured by the SAS following individualized neurofeedback treatment.

The secondary research questions posed in the present study aimed to explore whether self-reported anxiety differed over time, specifically from pre to post, pre to follow-up, and post to follow-up. Interestingly, there is no significant change from post to follow-up SAS scores. This lack of change from post to follow-up anxiety scores imply neurofeedback treatment for one university academic semester has lasting effects from post to follow-up, or around a month between time points. Our findings are comparable to results of a similar study examining post and follow-up by Van Doren et al. (2019). Their neurofeedback study for ADHD reported no significant change from post to follow-up. Additionally, the researchers stated that neurofeedback seems to be sustainable after 2 to 12 months. Our findings suggest sustainable results after 1 to 1.5 months. Similar to prior neurofeedback research, these findings indicate that neurofeedback is a sustainable and effective treatment for anxiety symptoms.

\section{Limitations and Implications for Research}

The lack of a control group is a major limitation of the study. As such, there is no way to determine what aspects of the results may be attributable to placebo effect. The individualized treatment protocolsbased on $q E E G$ results-created a variability in the treatment provided, with no analysis of whether specific protocols may have had differing effects. The smaller sample size of the primary data set and the small sample size of the secondary data set are another limiting factor for assessing statistical robustness. In addition, a number of issues are present due to the academic setting in which the research was conducted. Restricting the number of sessions to an academic semester reduced the possible number of sessions, which may have limited the effectiveness of treatment. The skill level of the student and volunteer clinicians may have varied somewhat and was not controlled for. As a result, there is no way to determine if more experienced clinicians may have had a higher level of treatment effectiveness. Finally, there was no control for the variability of other forms of treatment the subject may have received before or during the study, the result being a lack of differentiation between the effects of the study and the effects of other treatments.

The study supports the need for further research on the possible efficacy of neurofeedback for the amelioration of anxiety symptoms in a number of areas. Identifying biomarkers of anxiety in the EEG is a possible focus of future studies by comparing the qEEG and other analyses of EEG data for subjects with or without anxiety symptoms. For example, in addition to mere amplitude measures, characteristics of coherence, independent components, network hubs, event-related potentials, dynamic timefrequency analysis, and source density location may be utilized.

\section{Conclusion}

This retrospective study included data from qEEG, individualized neurofeedback treatment protocols for anxiety. After running regression analyses, the results yielded three predictors of posttreatment outcomes: total problems, substance abuse mean, and education level. Further, an analysis of a subsample of data displayed statistically significant improvement from pre to post with sustainable outcomes from post to follow-up.

\section{Author Note}

This study was financially supported by the Foundation for Neurofeedback and Neuromodulation Research.

\section{Author Disclosure}

Authors have no other grants, financial interests, or conflicts to disclose.

\section{References}

American Psychiatric Association. (2013). Diagnostic and statistical manual of mental disorders (5th ed.). Washington, DC: Author.

Achenbach, T. M., \& Rescorla, L. A. (2003). Manual for the ASEBA adult forms and profiles. Burlington, VT: University of Vermont, Research Center for Children, Youth, \& Families.

Arns, M., Drinkenburg, W., \& Kenemans, J. L. (2012). The effects of QEEG-informed neurofeedback in ADHD: An open-label pilot study. Applied Psychophysiology and Biofeedback, 37, 171-180. https://doi.org/10.1007/s10484-012-9191-4

Arns, M., Heinrich, H., \& Strehl, U. (2014). Evaluation of neurofeedback in ADHD: The long and winding road. Biological Psychology, 95, 108-115. https://doi.org/10.1016 /j.biopsycho.2013.11.013 
Busner, J., \& Targum, S. D. (2007). The clinical global impressions scale: Applying a research tool in clinical practice. Psychiatry (Edgmont), 4(7), 28-37. Retrieved from http://search.proquest.com/docview/733162476/

Cheon, E.-J., Koo, B.-H., Seo, W.-S., Lee, J.-Y., Choi, J.-H., \& Song, S.-H. (2015). Effects of neurofeedback on adult patients with psychiatric disorders in a naturalistic setting. Applied Psychophysiology and Biofeedback, 40(1), 17-24. https://doi.org/10.1007/s10484-015-9269-x

Demerdzieva, A., \& Pop-Jordanova, N. (2011). Alpha asymmetry in QEEG recordings in young patients with anxiety. Prilozi / Makedonska Akademija Na Naukite i Umetnostite, Oddelenie Za Biološki i Medicinski Nauki = Contributions / Macedonian Academy of Sciences and Arts, Section of Biological and Medical Sciences, 32(1), 229-244.

Gunkelman, J. (2006). Transcend the DSM using phenotypes. Biofeedback, 34(3), 95-98. Retrieved from https://www.aapb.org/files/publications/biofeedback/2006/biof 34_3_DSM.pdf

Hammond, D. C. (2010). The need for individualization in neurofeedback: Heterogeneity in QEEG patterns associated with diagnoses and symptoms. Applied Psychophysiology and Biofeedback, 35(1), 31-36. https://doi.org/10.1007 /s10484009-9106-1

Heller, W., Nitschke, J. B., Etienne, M. A., \& Miller, G. A. (1997). Patterns of regional brain activity differentiate types of anxiety. Journal of Abnormal Psychology, 106(3), 376-385. https://doi.org/10.1037/0021-843X.106.3.376

Hill, R. W. \& Castro, E. (2002). Getting rid of Ritalin: How neurofeedback can successfully treat attention deficit disorder without drugs. Charlottesville, VA: Hampton Roads.

Jones, M. S. (2015). Comparing DC offset and impedance readings in the assessment of electrode connection quality. NeuroRegulation, 2(1), 29-36. https://doi.org/10.15540 /nr.2.1.29

Jones, M. S., \& Hitsman, H. (2018). QEEG-guided neurofeedback treatment for anxiety symptoms. NeuroRegulation, 5(3), 8592. https://doi.org/10.15540/nr.5.3.85

Kerson, C., Sherman, R. A., \& Kozlowski, G. P. (2009). Alpha suppression and symmetry training for generalized anxiety symptoms. Journal of Neurotherapy, 13(3), 146-155. https://doi.org/10.1080/10874200903107405

Krigbaum, G. \& Wigton, N. L. (2014) When discussing neurofeedback, does modality matter? NeuroRegulation. 1(1), 48-60. https://doi.org/10.15540/nr.1.1.48

National Institute of Mental Health. (2017). Prevalence of any anxiety disorder among adults. Retrieved from https://www.nimh.nih.gov/health/statistics/any-anxietydisorder.shtml\#part 155094

National Institute of Mental Health. (2018). Anxiety disorders. Retrieved from http://www.nimh.nih.gov/health/topics/anxietydisorders/index.shtm

National Institute of Mental Health. (2018). Anxiety disorders. Signs and symptoms. Retrieved from https://www.nimh.nih.gov /health/topics/anxiety-disorders /index.shtml\#part 145336

Ogrim, G., \& Hestad, K. A. (2013). Effects of neurofeedback versus stimulant medication in attention-deficit/hyperactivity disorder: A randomized pilot study. Journal of Child and Adolescent Psychopharmacology, 23(7), 448-457. https://doi.org/10.1089 /cap.2012.0090

Price, J., \& Budzynski, T. (2009). Anxiety, EEG patterns, and neurofeedback. In T. H. Budzynski, H. K. Budzynski, J. R. Evans, \& A. Abarbanel (Eds.), Introduction to quantitative EEG and neurofeedback: Advanced theory and applications (2nd ed., pp. 453-472). Burlington, MA: Elsevier Academic Press. https://doi.org/10.1016/B978-0-12-374534-7.00017-4

Stern, J. M. (2005). Atlas of EEG patterns. Philadelphia, PA: Lippincott Williams \& Wilkins.

Scheinost, D., Stoica, T., Saksa, J., Papademetris, X., Constable, R. T., Pittenger, C., \& Hampson, M. (2013). Orbitofrontal cortex neurofeedback produces lasting changes in contamination anxiety and resting-state connectivity. Translational Psychiatry, 3(4), e250. https://doi.org/10.1038/tp.2013.24

Tanaka-Matsumi, J., \& Kameoka, V. A. (1986). Reliabilities and concurrent validities of popular self-report measures of depression, anxiety, and social desirability. Journal of Consultation and Clinical Psychology, 54(3), 328-333. https://doi.org/10.1037/0022-006X.54.3.328

Tharawadeepimuk, K., \& Wongsawat, Y. (2014, November). QEEG evaluation for anxiety level analysis in athletes. Paper presented at the 2014 7th Biomedical Engineering International Conference (BMEiCON 2014), Fukuoka, Japan (pp. 172-175). Piscataway, NJ: IEEE. https://doi.org/10.1109 /BMEiCON.2014.7017400

Van Doren, J., Arns, M., Heinrich, H., Vollebregt, M. A., Strehl, U., \& Loo, S. K. (2019). Sustained effects of neurofeedback in ADHD: A systematic review and meta-analysis. European Child \& Adolescent Psychiatry, 28(3), 293-305. https://doi.org/10.1007/s00787-018-1121-4

Wang, S.-Y., Lin, I.-M., Fan, S.-Y., Tsai, Y.-C., Yen, C.-F., Yeh, Y.C., ... Lin, H.-C. (2019). The effects of alpha asymmetry and high-beta down-training neurofeedback for patients with the major depressive disorder and anxiety symptoms. Journal of Affective Disorders, 257, 287-296. https://doi.org/10.1016 /j.jad.2019.07.026

Walker, J. E. (2012). Remediation of enuresis using QEEG-guided neurofeedback training. Biofeedback, 40(3), 109-112. https://doi.org/10.5298/1081-5937-40.3.04

White, E. K., Groeneveld, K. M., Tittle, R. K., Bolhuis, N. A., Martin, R. E., Royer, T. G., \& Fotuhi, M. (2017). Combined neurofeedback and heart rate variability training for individuals with symptoms of anxiety and depression: A retrospective study. NeuroRegulation, 4(1), 37-55. https://doi.org/10.15540 /nr.4.1.37

Wigton, N. L. (2014). Evaluating 19-channel z-score neurofeedback: Addressing efficacy in a clinical setting (Doctoral dissertation). Available from ProQuest Dissertations and Theses database. (UMI No. 3625170)

Wigton, N. L., \& Krigbaum, G. (2015). Attention, executive function, behavior, and electrocortical function, significantly improved with 19-channel $z$-score neurofeedback in a clinical setting: A pilot study. Journal of Attention Disorders. Advance online publication. https://doi.org/10.1177/1087054715577135

Zhao, Z., Yao, S., Li, K., Sindermann, C., Zhou, F., Zhao, W., ... Becker, B. (2018). Real-time functional connectivity-based neurofeedback of amygdala-frontal pathways reduces anxiety. BioRxiv. Advance online publication (preprint). https://doi.org $/ 10.1101 / 308924$

Zung, W. W. K. (1971). A rating instrument for anxiety disorders. Psychosomatics, 12(6), 371-179. https://doi.org/10.1016 /S0033-3182(71)71479-0

Received: March 7, 2020

Accepted: March 20, 2020

Published: March 25, 2020 